\title{
Modelling Chloride Penetration in Concrete Using Electrical Voltage and Current Approaches
}

\author{
Juan Lizarazo-Marriaga ${ }^{a^{*}}$, Peter Claisse $e^{\mathrm{b}}$ \\ ${ }^{a}$ Departamento de Ingeniería Civil, Universidad Nacional, Bogotá, Colombia \\ ${ }^{\mathrm{b}}$ Construction Materials Applied Research Group, Coventry University CVI 5FB, UK
}

Received: August 5, 2010; Revised: February 4, 2011

\begin{abstract}
This paper reports a research programme aimed at giving a better understanding of the phenomena involved in the chloride penetration in cement-based materials. The general approach used was to solve the Nernst-Planck equation numerically for two physical ideal states that define the possible conditions under which chlorides will move through concrete. These conditions are named in this paper as voltage control and current control. For each condition, experiments and simulations were carried out in order to establish the importance of electrical variables such as voltage and current in modelling chloride transport in concrete. The results of experiments and simulations showed that if those electrical variables are included as key parameters in the modelling of chloride penetration through concrete, a better understanding of this complex phenomenon can be obtained.
\end{abstract}

Keywords: chloride penetration, electrical migration tests, concrete

\section{Introduction}

The durability of concrete is of primary concern to the engineering community due to the substantial cost of repairing ageing infrastructure. In the majority of cases, weakness and structural breakdown can be directly attributed to damage due to steel corrosion. As the presence of chloride ions heightens the level of metallic corrosion activity, the mechanisms by which such chlorides penetrate the concrete are of great interest.

Despite the large amount of research and computer modelling that has taken place, simplified models for explaining chloride penetration in the concrete are still commonly in use. Fick's Laws remain in widespread and popular use where a simple approach is thought desirable. Indeed most of the established concrete diffusion models are based around the analytical solution of Fick's Second Law. Although great advances have been made in these models, and it is common to find models using time-dependent chloride diffusion coefficients or time-dependent chloride binding isotherms ${ }^{1}$, Fick's Laws do assume that chlorides are isolated particles. This assumption is not an entirely realistic one, because in the pore solution of concrete, chloride ions will typically be interacting with other ions according to their electrical charge.

One of the reasons why Fick's Laws have not been completely replaced by other more "appropriate" theories or models is because according to them, the chloride penetration depends entirely on a single parameter, the coefficient of diffusion. To define a complex phenomenon using a single coefficient is a situation that brings several advantages to engineers; however, scientists have known for some time that the Nernst-Planck equation ${ }^{2}$ should be used to account for possible ionic interactions.

The Nernst-Planck equation includes an electrical field in addition to a gradient of ionic concentration, so it has been proposed as a tool to determine the chloride diffusion coefficient in experimental migration tests as the NTBuild- $492^{3}$. However, using those approaches the Nernst-Planck model continues to be used assuming the chloride ions are isolated from other species.
Multi-species models have been developed in recent years ${ }^{2,4}$. These incorporate the presence of several free species in the pore solution (normally $\mathrm{OH}^{-}, \mathrm{Na}^{+}$, and $\mathrm{K}^{+}$) along with the chlorides penetrating from the exterior. Although the Nernst-Planck equation governs the phenomenon, it does need to be coupled with the physical principle of charge neutrality, which must be preserved at any point. Even in a self diffusion test, the condition of charge neutrality generates an electric field which affects the flow of each ion. For a multi-ionic system the major difficulty is the determination of those parameters that will define the transport (principally the diffusion coefficients) of all the species involved.

This paper reports a research programme aimed at giving a better understanding of the phenomena involved in the chloride penetration in cement-based materials. The general approach used was to solve the Nernst-Planck equation numerically for two physical ideal states that define the possible conditions under which chlorides will move through concrete. These conditions are named in this paper as voltage and current control. For each condition, simulations were carried out in order to establish the importance of electrical variables such as voltage and current.

\section{Modelling-Theoretical Background}

\subsection{Voltage control}

The first condition called "voltage control" is defined by a concrete sample placed between two electrodes that apply a constant potential difference between them and the transient current across the sample is measured. Under this condition a migration test like the ASTM- C $1202^{5}$ or NTBuid-492 can be simulated. The model used in this paper uses a one-dimensional finite difference scheme (divided by space and time steps) to solve the Nernst-Planck equation (Equation 1) that can describe simultaneously the flux of several ionic species under possible driving forces such as diffusion (concentration gradient) and migration (electrical field) ${ }^{6}$. 


$$
J_{i}=-D_{i} \frac{\partial C_{i}}{\partial x}-\frac{z_{i} F}{R T} D_{i} C_{i} \frac{\partial E}{\partial x}
$$

$J_{\mathrm{i}}$ is the flux of species $i, D_{\mathrm{i}}$ is the diffusion coefficient of species $i$, $C_{\mathrm{i}}$ is the ionic concentration of species $i$ in the pore fluid, and $x$ is the distance, where $z_{\mathrm{i}}$ is electrical charge of specie $i, F$ is the Faraday constant, $R$ is the gas constant, $T$ the absolute temperature and $E$ the electrical voltage.

In the electro-diffusion model, the concentration of ions is defined in two ways: the free ions per unit volume of liquid $\left(C_{1}\right)$ and the total ions per unit volume of solid $\left(C_{\mathrm{s}}\right)$. The ratio of those concentrations is named the factor binding capacity $(\alpha)$. In the same way, the intrinsic coefficient $\left(D_{\mathrm{i}}\right)$ defines the transport of matter when the flux is calculated per unit cross-sectional area of the pores and the concentration in the free liquid; and, the apparent diffusion coefficient $\left(D_{\text {app }}\right)$ defines the transport of any ion when the flux is calculated per unit area of the porous material and the average concentration in the material, Equation 2. For chlorides, the binding chloride capacity in the model used a linear isotherm, in contrast, for other species was assumed no binding or adsorption with the cement matrix. By integrating the definitions given above it is possible to prove that the ratio chloride intrinsic diffusion to apparent diffusion coefficient is equal to the ratio binding factor capacity to porosity.

$$
\frac{\alpha}{\varepsilon}=\frac{D_{C l}}{D_{a p p-c l}}
$$

During the numerical process, in the model for any time step the fluxes and the changes of concentration are calculated for all the species involved; however, the model ensures that charge neutrality is maintained throughout the sample at all times for all the ions together. If there is any charge build-up the model corrects the actual migration flux until the charge balance is within a specified range. Macroscopically, the procedure to maintain the charge balance is according to Kirchoff's law: "...the current density ( $i$ ) into any point will equal the current out of it". That means that for any time the total current in node $i$ is equal to the current in node $i+1$, Equation 3.

$$
\sum_{k} i_{i, k}^{t}=\sum_{k} i_{i+1, k}^{t}
$$

In a concrete chloride diffusion or migration test, the charge buildup generated by differences of mobility of ions in the pore solution is dissipated by the distortion of the electrical field. Those effects of voltage changing are applied in the model by distorting iteratively the voltage in each space step removing any build-up of charge. In that way, only a multi-species system can be physically possible because if there is only one ion the charge neutrality never is reached.

That voltage distortion is not just an artificial or numerical mechanism, it is a real physical phenomenon measured and reported previously by Lizarazo-Marriaga and Claisse ${ }^{7}$, who called it nonlinear membrane potential due the physical similarities with the liquid-junction potential produced in a porous membrane ${ }^{8}$. Whenever different ions with different mobilities at different concentrations in the concrete pore solution start to move, there is a tendency for the segregation of charge and the breakdown of the law of electro-neutrality. As a result, several complex and not completely well understood interactions happen in the pore solution, such as the interaction of ions with the solvent or the electrical ion-ion interactions. In order to quantify the ion-ion interactions the more acceptable and used electrochemical theory was that proposed by Debye and Hückel in 1923.

From a "simplistic" microscopic point of view, the Debye and Hückel (ion-cloud) theory can be used to calculate the electrostatic potential contributed by the surrounding ions to the total electric potential at one reference ion. Once a reference ion is selected, the solvent molecules are replaced by a dielectric continuum medium $(\varepsilon)$ and the other ions involved are replaced by an excess of charge density (Figure 1). Assuming that the excess charge distribution is spherically symmetrical around the reference ion, the relationship between excess charge density $\left(\rho_{\mathrm{r}}\right)$ and electrostatic potential $\left(\psi_{\mathrm{r}}\right)$ at a specific radial distance $(r)$ can be given through Poisson's equation ${ }^{8}$, Equation 4.

$$
\frac{1}{r^{2}} \frac{d}{d r}\left(r^{2} \frac{d \psi_{r}}{d r}\right)=-\frac{4 \pi}{\varepsilon} \rho_{r}
$$

The excess charge density in the volume element $d V$ is obtained in the Debye-Hukel theory through the linearization of the Boltzmann equation $^{8}$, Equation 5.

$$
\rho_{r}=-\sum_{i} \frac{n_{i}^{o} z_{i}^{2} e_{o}^{2}}{k T} \psi_{r}
$$

$e_{0}$ is the electronic charge, $n_{\mathrm{i}}$ is the total number of ions $i$ per unit volume, $k$ is the Boltzmann constant, and the $\Sigma$ symbol refers to all the species $i$. Similarly, the maximum value of charge in a spherical cloud is given through the Debye Huckel length $\left(\kappa^{-1}\right)$, which give an idea about the thickness of the ionic atmosphere, Equation 6.

$$
\kappa^{2}=\frac{4 \pi}{\varepsilon k T} \sum_{i} n_{i}^{o} z_{i}^{2} e_{o}^{2}
$$

So, the problem can be simplified and linearized and a general equation can be obtained, Equation 7.

$$
\frac{1}{r^{2}} \frac{d}{d r}\left(r^{2} \frac{d \psi_{r}}{d r}\right)=\kappa^{2} \psi_{r}
$$

The solution of which is shown as Equation 8 .

$$
\psi_{r}=\frac{z_{i} e_{O}}{\varepsilon} \frac{e^{-\kappa r}}{r}
$$

It can be seen from Equation 8 that the microscopic treatment of the electrostatic potential for the ion-ion interaction has a huge complexity. It has been defined for the reference ion as a matter of study; however, it needs to be applied to the whole concrete system.

Going from a micro to a macro approach, a general model with a bigger scale allows a similar, but simpler solution. The simple treatment of the concrete as a circuit in which physical laws must be valid seems to be more appropriate. In that way, the macroscopic electrical model introduced in this paper permits the inclusion of the ion-ion effects coupling all the species involved, to the charge neutrality.

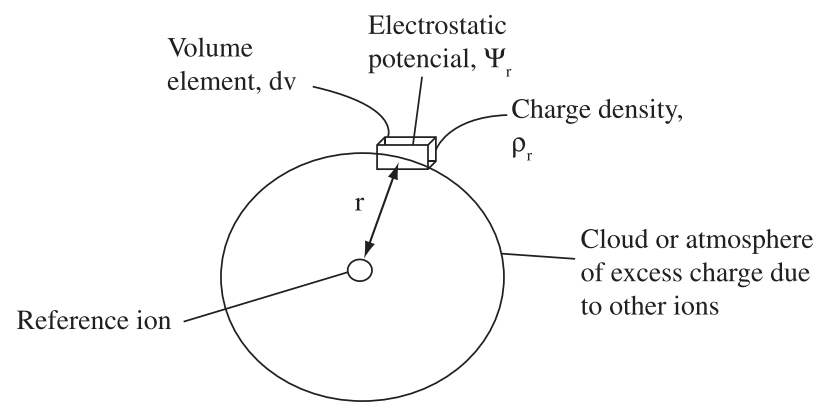

Figure 1. Ionic cloud of an electrical charged element ${ }^{8}$. 


\section{Current Control}

In the second condition called "current control" the concrete between two electrodes is subject to a constant electrical current. For this condition, when the current is zero, the phenomenon of normal diffusion with no applied potential can be simulated. If the "current control" condition is used with a non-zero current, the phenomena of either Cathodic protection or electrochemical chloride removal can be modelled.

It is of note that theoretically, when chloride ions are just permitted to diffuse through concrete the system acts like a dry-cell battery and a voltage is developed. Some previous authors have modelled chloride diffusion by simply integrating Fick's Law and using a diffusion coefficient for chloride ions. The true situation in normal diffusion is, however, far more complex because the voltage that is developed will inhibit further transport unless it is dissipated by further ionic transport. This further transport may be negative ions (such as $\mathrm{OH}^{-}$) moving in the opposite direction to the $\mathrm{Cl}^{-}$or positive ions (such as $\mathrm{Na}^{+}$) moving with them and may be the determining factor for the rate of transport of the $\mathrm{Cl}^{-}$. This should not be modelled with a voltage control algorithm set to zero applied voltage because this implies that the two ends of the sample are short-circuited.

In the numerical algorithm used with a non-zero current control condition, the physical equations used to avoid the charge build-up are the same used previously (voltage control); however, in this approach, in addition to keeping the charge neutrality throughout the sample at all times for all the ions together, it is necessary to keep the current density constant during whole duration of the test. Figure 2 shows a schematic representation of the electrical voltage and current conditions.

\section{Experimental Programme}

\subsection{Materials}

A single mortar mixture having a water to cement ratio of 0.36 and a sand-to-binder ratio of 3 was cast. Ordinary Portland cement
CEM I without mineral or chemical admixtures was used. All the specimens were cured under lime water at $21 \pm 2{ }^{\circ} \mathrm{C}$ prior to testing for 90 days. Because of the low water to cement ratio the mixture had a poor workability, it was compacted manually using prolonged tamping in order to reach its maximum density. Table 1 shows the proportions of the materials used.

\subsection{Test methods}

Experimental voltage control tests were carried out to ASTM-C1202. Using this standard, a steady external electrical potential of 60 volts D.C was applied to a sample of concrete of $50 \mathrm{~mm}$ thick and $100 \mathrm{~mm}$ diameter for 6 hours. The anode and cathode were filled with $0.30 \mathrm{~N}$ sodium hydroxide and $3.0 \%$ sodium chloride solutions, respectively. These samples were prepared before the test in conformity with the ASTM standard. Additionally, the voltage membrane potential was calculated using a salt bridge in a drilled hole in the top of the sample giving readings of the actual voltage in the sample during the test (Figure 3 ). These readings confirmed that the voltage drop across the sample is not linear and varies with time ${ }^{10,11}$.

\subsection{Calculation of transport properties}

The chloride related transport properties needed to simulate the chloride effects on a voltage or current control test for the mixture proposed were obtained from the experimental voltage control results by feeding an artificial neural network with results from the electrochemical model ${ }^{10,11}$. Table 2 is a summary of the principal transport properties and variables obtained following that methodology. The heat loss factor is a property of the cell and is adjusted to give the observed temperature rise due to the modelled Joule heating in the sample.

Table 1. Mortar mixture proportions.

\begin{tabular}{cccc}
\hline $\begin{array}{c}\text { Water-to- } \\
\text { binder ratio }\end{array}$ & $\begin{array}{c}\text { OPC } \\
\left(\mathrm{kg} \cdot \mathrm{m}^{-3}\right)\end{array}$ & $\begin{array}{c}\text { Sand } \\
\left(\mathrm{kg} \cdot \mathrm{m}^{-3}\right)\end{array}$ & $\begin{array}{c}\text { Water } \\
\left(\mathrm{kg} \cdot \mathrm{m}^{-3}\right)\end{array}$ \\
\hline 0.36 & 650 & 1950 & 234 \\
\hline
\end{tabular}

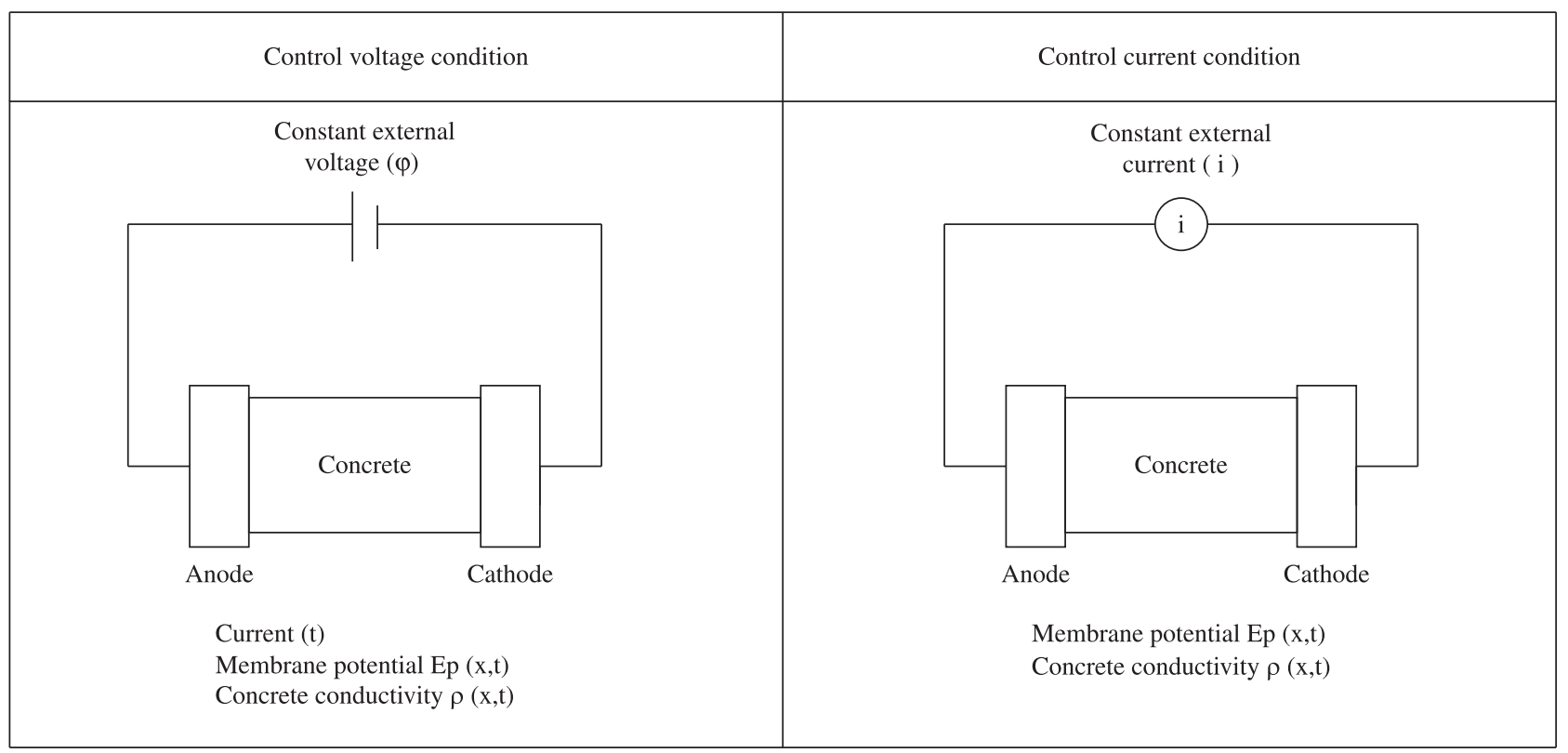

Figure 2. Electrical voltage and current approaches ${ }^{9}$. 


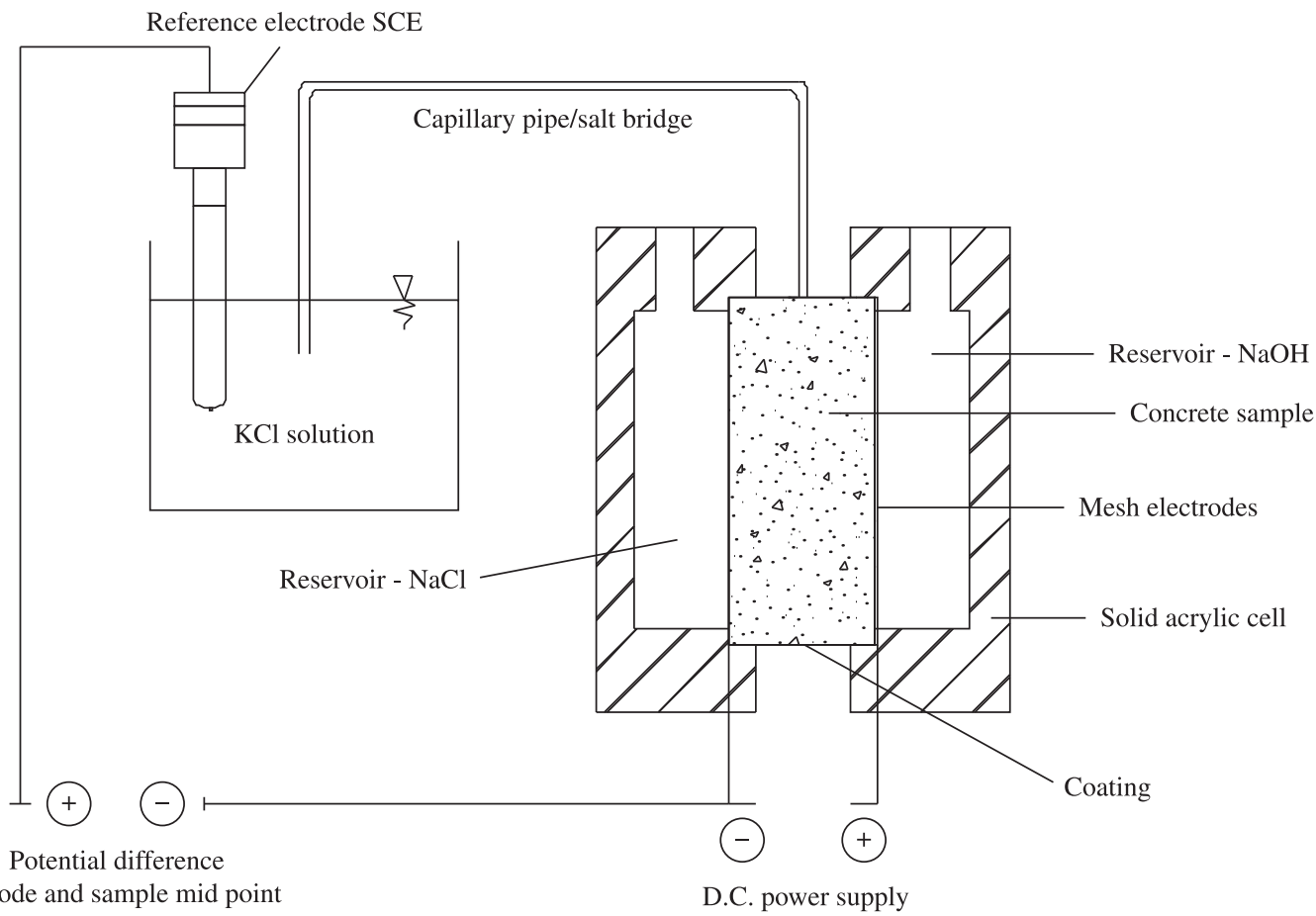

Figure 3. ASTM C1202 test and salt bridge used to measure the membrane potential.

Table 2. Optimized and physical parameters used in the simulations.

\begin{tabular}{|c|c|c|c|c|c|c|}
\hline & \multirow{3}{*}{$\begin{array}{l}\text { Valence } \\
\text { (Z) }\end{array}$} & \multirow{3}{*}{$\begin{array}{c}\text { Intrinsic } \\
\text { Diffusion coefficient } \\
\mathrm{D}\left(\mathrm{m}^{2} / \mathrm{s}\right)\end{array}$} & \multirow{2}{*}{\multicolumn{3}{|c|}{$\begin{array}{c}\text { Concentration } \\
\text { (in liquid) }\left(\mathrm{mol} . \mathrm{m}^{-3}\right)\end{array}$}} & \multirow{3}{*}{$\begin{array}{c}\text { Binding capacity } \\
\text { factor }\end{array}$} \\
\hline & & & & & & \\
\hline & & & Negative & In sample & Positive & \\
\hline Hydroxyl ion & -1 & 7.877E-11 & 0 & 239.973 & 300 & 0.171 \\
\hline Chloride ion & -1 & $1.904 \mathrm{E}-10$ & 530 & $0.00 \mathrm{E}+00$ & 0 & 0.383 \\
\hline Sodium ion & 1 & $2.061 \mathrm{E}-11$ & 530 & $7.92 \mathrm{E}+01$ & 300 & 0.171 \\
\hline Potassium ion & 1 & $4.099 \mathrm{E}-12$ & 0 & $1.61 \mathrm{E}+02$ & 0 & 0.171 \\
\hline No of cells & 100 & & Sampl & ngth (m) & 0.05 & \\
\hline Time step (seconds) & 2 & & Sampl & dius $(m)$ & 0.05 & \\
\hline Run time (hours) & 6 & & Room te & rature $(\mathrm{K})$ & 295 & \\
\hline Porosity & 0.171 & & Cell v & nes $\left(m^{3}\right)$ & $2.00 \mathrm{E}-04$ & \\
\hline Applied voltage (V) & 60 & & Heat los: & tor $\left(\mathrm{J} . \mathrm{K}^{-1}\right)$ & 0.30 & \\
\hline
\end{tabular}

\section{Computer Simulations}

Using the transport related properties of the optimized mortar (Table 2); the voltage control ASTM C-1202 test was simulated. Additionally, using the same setup and tests conditions, current control tests were simulated under conditions of non-zero current (0.1 $\mathrm{A}-6$ hours) and zero current $(0 \mathrm{~A}-12$ days $)$.

\section{Results and Discussion}

\subsection{Voltage control}

The simulated membrane potential for the mortar mixture is shown in Figure 4 as a colour contour map. It can be observed that the membrane potential is non-linear in time and position and only during the initial time step at the start of the test can the electrical field be considered linear. If the model is used without the voltage correction, (i.e., no charge neutrality and no coupling among species), the membrane potential would have a value of zero for each time and position.

Figures 5 and 6 show the ionic current carried for anions and cations included in the simulation. Although the total current at any point within the sample should be constant for a specific time, the currents passed by individual ions do not follow this rule and are typically different. From the figures it can be observed that the hydroxide and chloride ions are responsible for most of the total current passed. At the beginning of the test, hydroxyl ions predominate; however, around two hours later, chlorides, which are now penetrating massively into the sample, have begun to control the current being passed. By the end of the test, the chloride ions were responsible for about $80 \%$ of the total current. The role of cations 
is less important than that of anions; however, they are essential for establishing the necessary conditions of neutrality within the system. Although chloride and hydroxide are much more mobile species, without sodium and potassium the neutrality condition would never be successfully achieved.

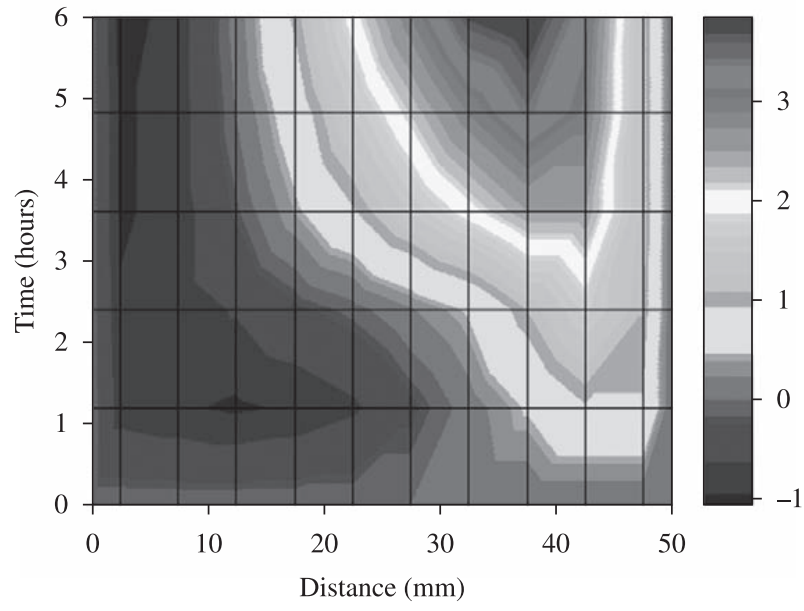

Figure 4. Membrane potential (V) for the mortar mixture.

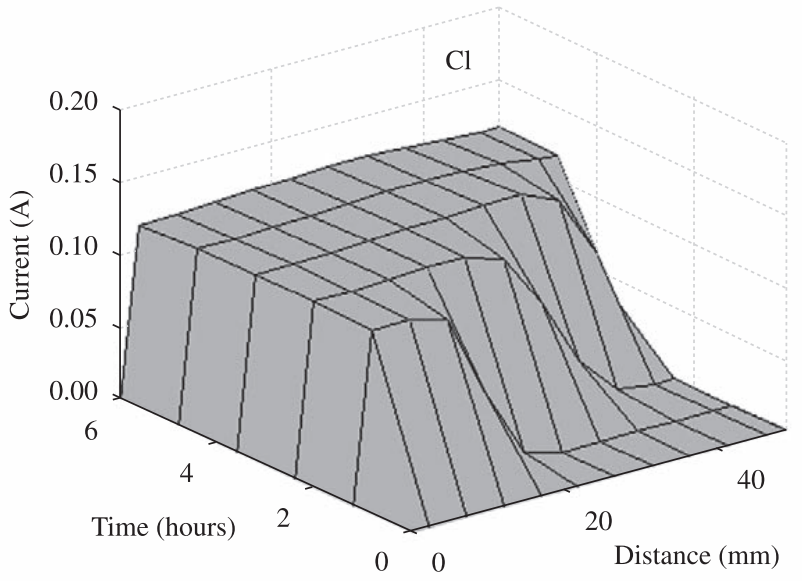

Figure 7 shows the simulated current passed for each ion and the total current for three different times of the test. Figure 7a shows the condition where no voltage correction is taken into account in the model, so the condition of charge neutrality is not satisfied (no membrane potential or no ionic coupling). Figure $7 \mathrm{~b}$ shows similar results using the voltage correction included in the model, where it can be seen that for the same time, the total current in all the length of the sample is the same; however, the contribution of each ion is not necessarily the same.

For the mortar sample simulated, the ionic concentrations of $\mathrm{OH}^{-}, \mathrm{Cl}^{-}, \mathrm{Na}^{+}$, and $\mathrm{K}^{+}$are shown in Figure 8 as colour contour maps. At the beginning of the test, chlorides were present only in the cathode external reservoir; however, as the test progressed, chlorides penetrated the specimen and reached the other external cell (anode). Also, from the results of concentrations, it can be seen that prior to the start of the test, the hydroxyl ions were uniformly distributed in the pore solution and in the anode external cell. However, the concentration of those ions increases in the cathode while decreasing in the anode. This behaviour is considered realistic because in an actual test the hydroxyl ions will migrate towards the anode where they are oxidized, releasing electrons. Simultaneously, at the cathode, hydroxyl ions and oxygen are released from water molecules, as a result of electrolysis. The results presented agree well with the findings of Prince, Perami, and Espagne ${ }^{12}$.

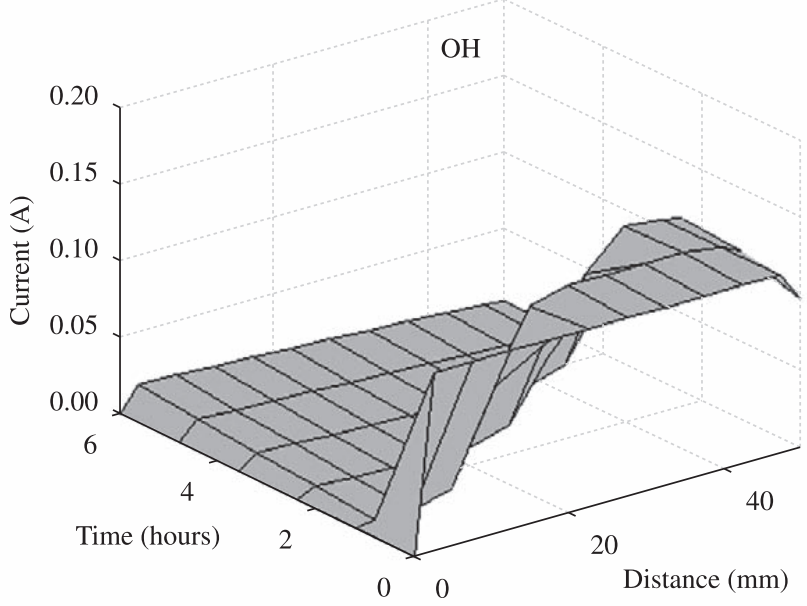

Figure 5. Ionic current during the ASTM C1202 test: chloride (left) and hydroxyl ions (right).
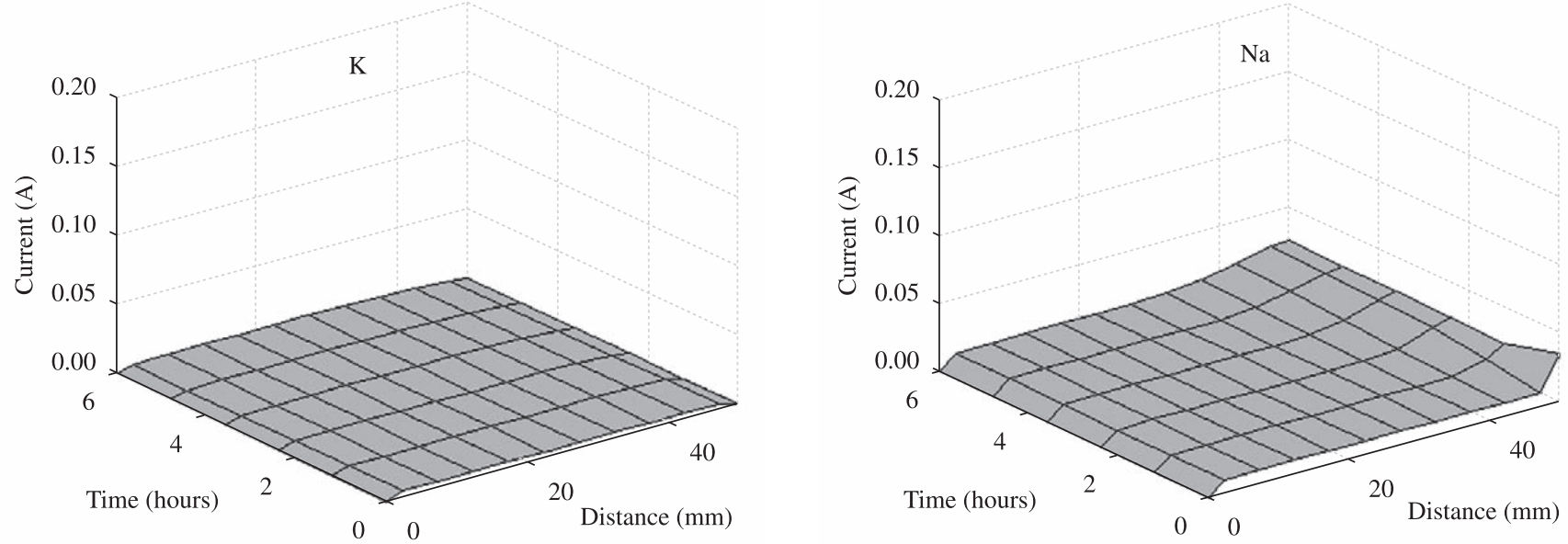

Figure 6. Ionic current during the ASTM C1202 test: potassium (left) and sodium (right). 


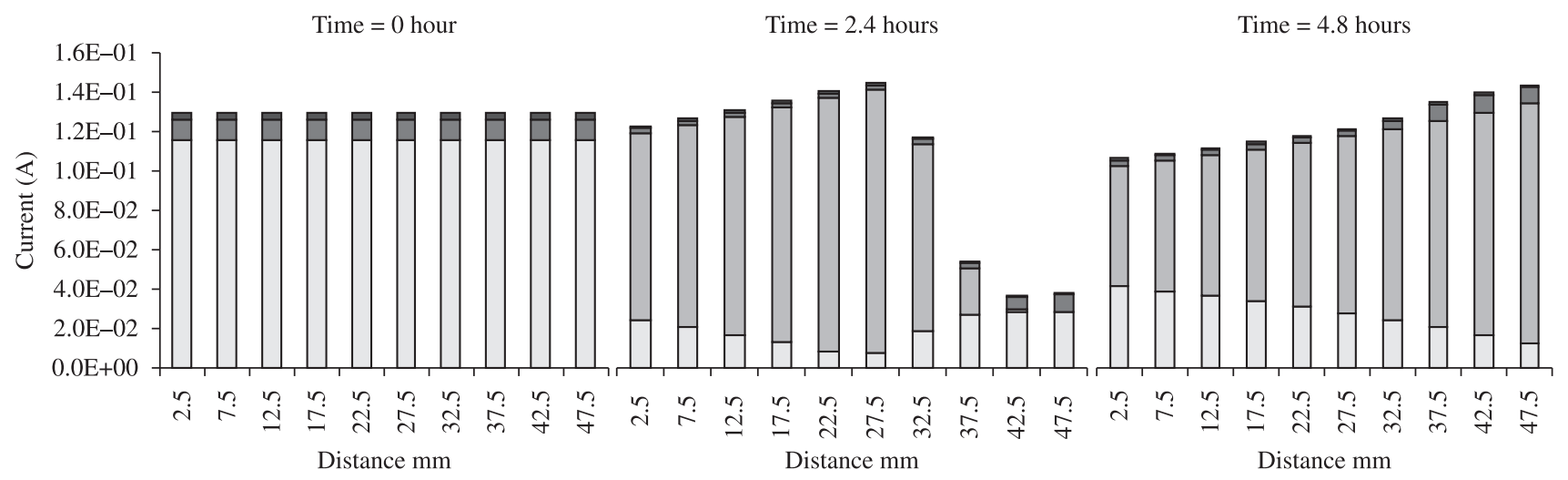

(a)

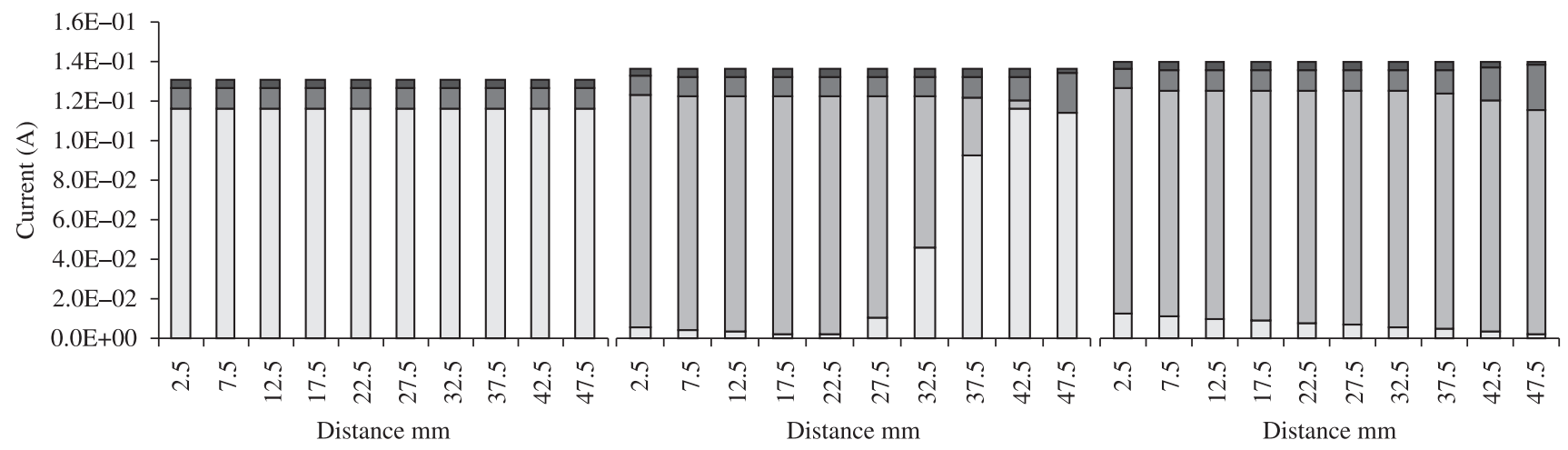

(b)

$\square$ Hydroxyl $\square$ Chloride $\square$ Sodium $\square$ Potassium

Figure 7. Ionic current in a ASTM C1202 test. a) No voltage correction (no ionic interaction); and b) voltage correction (ionic interaction).

\subsection{Current control model (non-zero current)}

Under a current control condition the concrete sample subjected to a steady known electrical current produces a corresponding external voltage that does not remain constant over time. The simulated voltage generated at different times, under a constant $100 \mathrm{~mA}$, is shown in Figure 9. At the beginning of the test, the voltage drop was linear through the sample; however, voltage deviations were necessary to keep the total current constant. Simulations of ionic current for each ion included in this simulation showed a similar behaviour to that obtained in Figures 5 and 6. Chloride and hydroxyl ions were responsible for the majority of the current carried, while sodium and potassium together carried only a minor amount of the overall current.

The free chloride concentration profile for the $100 \mathrm{~mA}$ current control test, simulated at different times, is shown in Figure 10. This test is regarded as a non-steady condition, the source reservoir is depleting over time and the chloride ions are just beginning to reach the anode reservoir.

\subsection{Current control model (zero current)}

This condition reflects a standard diffusion cell using the same cells and solutions stated in the standard ASTM C1202 test but with no applied voltage. Figure 11 shows the membrane potential results across the entire sample obtained from the numerical current and voltage control modes at the end of the test (12 days). In the voltage control model, the membrane potential across the whole sample, between anode and cathode $(50 \mathrm{~mm})$, was equal to zero. This was expected because the voltage control states an external voltage applied of $0 \mathrm{~V}$ (i.e. an electrical short circuit connecting the ends of the sample). In contrast, although the applied external current was zero, the current control model showed a value of membrane potential of $-0.03 \mathrm{~V}$ between anode and cathode.

Figure 12 shows the results of total current calculated at 12 days of diffusion for different modelling conditions. As was expected, using the current control model (CC), the total current simulated at different positions at the end of the test was always approximately equal to zero. In contrast, for the voltage control model (VC) with the applied voltage equal to zero, a small total current was developed $(0.00008 \mathrm{~A})$. It is important to notice that in both models, the charge neutrality condition was fulfilled, but in the current control condition it was forced to be equal to zero. In contrast, the simulations of the total current without coupling all the species involved (VC-no correction) give very high numerical values of current because the charge neutrality is not met in full. Under this last condition, the Nernst-Plank equation is solved independently for each ion and as the external voltage is equal to zero, the model at the end solves the Fick's equation for each ion.

The simulated chloride profile for the voltage and current control conditions at 12 days is shown in Figure 13. Although the membrane potential and the current obtained from both models were different, important differences were not found between the concentration 

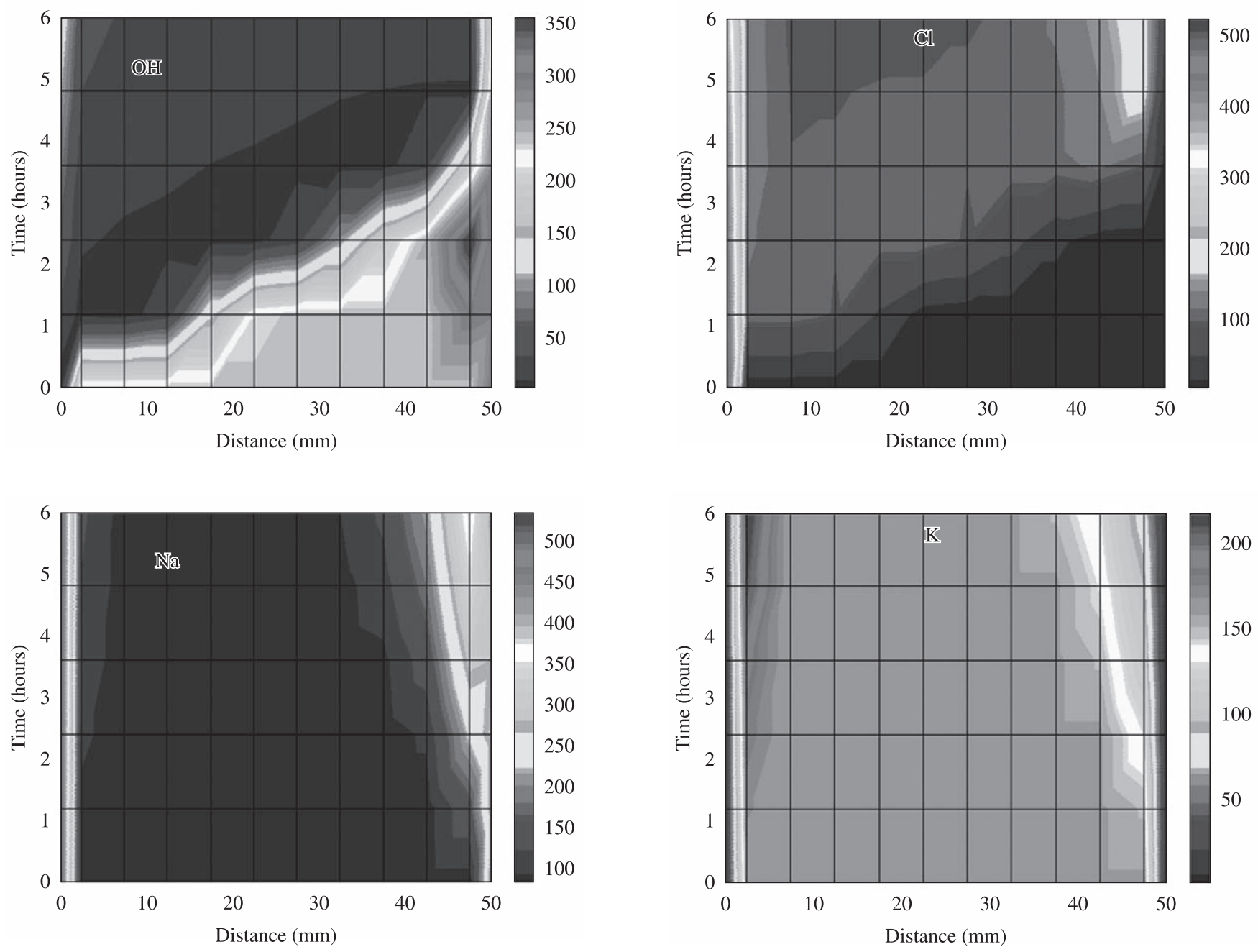

Figure 8. ASTM C-1202 concentrations for hydroxyl, chloride, sodium and potassium (mol.m-3).

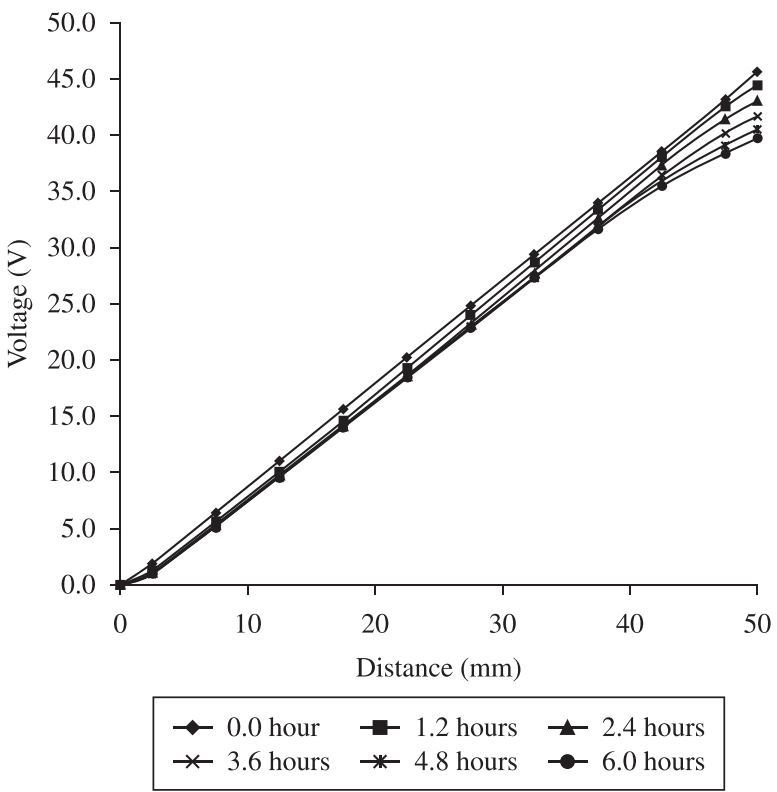

Figure 9. Voltage adjustments at different times $(100 \mathrm{~mA})$ during the current control condition.

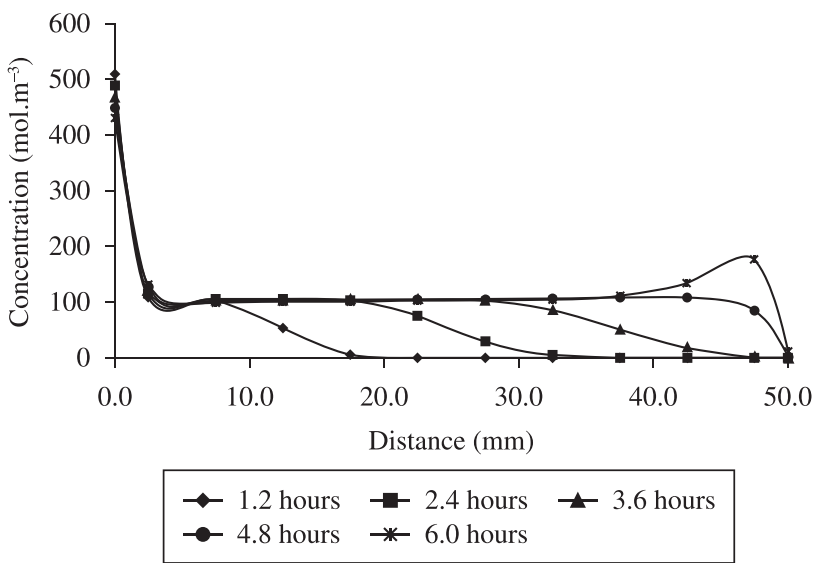

Figure 10. Chloride profile at different times during the current control condition $(100 \mathrm{~mA})$.

chloride profiles - the concentrations for both models at that specific time were very similar. In contrast, the profile obtained using the voltage control with no charge neutrality (i.e., Fick's law) is very different and a higher amount of chlorides are simulated. 


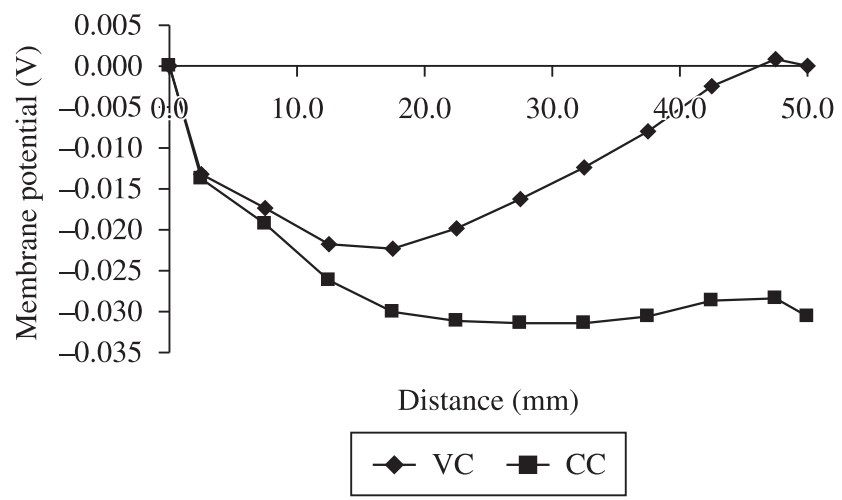

Figure 11. Membrane potential (respect to the cathode) at the end of the test (288 hours) current control: CC, and voltage control: VC.

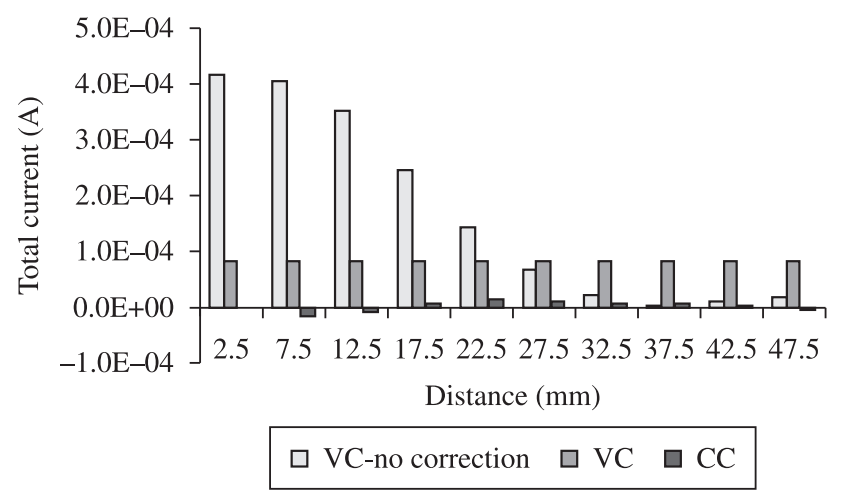

Figure 12. Total ionic current for different modelling assumptions: a) No ionic coupling (charge build-up); b) Voltage control, VC; and c) Current control, CC.

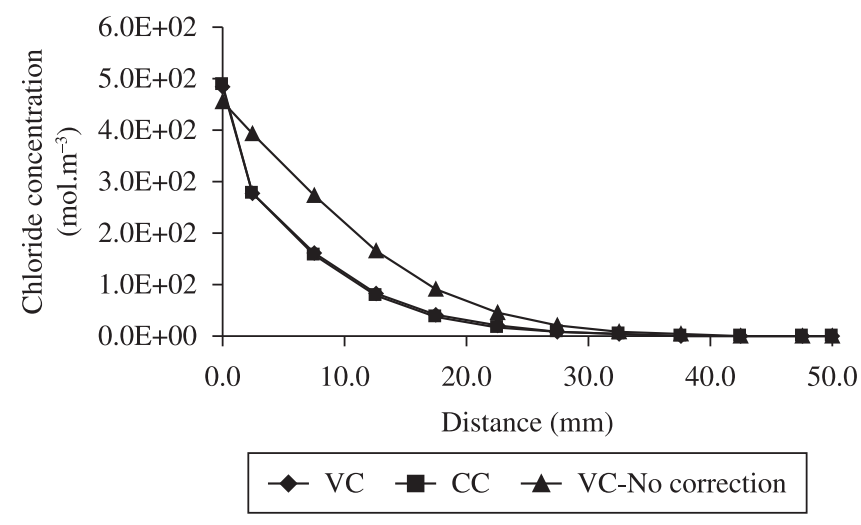

Figure 13. Chloride profiles obtained using different models.

\section{Conclusions}

The application of the Nernst-Planck equation to the simulation of diffusion or migration of any ionic species through a saturated porous medium using the non-linear voltage membrane potential should include all the microscopic interactions in a macroscopic way. The model proposed in this paper, coupled with the macroscopic application of Kirchhoff's circuit laws gives the possibility of calculating the individual flux for each of the involved ionic species.

The chloride penetration in cement-based materials can be simulated according to the physical conditions of the electrical equivalent circuit generated. In that way, using one of the three possible conditions introduced in this paper, is possible to simulate any real chloride penetration situation.

The measured current and the membrane potential voltage are key parameters for simulating ionic penetration through concrete.

\section{References}

1. Luping T. Engineering expression of the clinconc model for prediction of free and total chloride ingress in submerged marine concrete. Cement and Concrete Research. 2008; 38, (8-9)1092-1097.

2. Truc O, Ollivier JP and Nilsson LO. Numerical simulation of multi-species transport through saturated concrete during a migration test - Msdiff code. Cement and Concrete Research. 2000; 30(10):1581-1592.

3. NT-Build 492. Concrete, mortar and cement-based repair materials: chloride migration coefficient from non-steady-state migration experiments. Nordtest Method. Spoo, Finland: NORDTEST; 1999.

4. Claisse PA and Beresford TW. Obtaining more from the electrical chloride test. 1997; ACI - SP170-57 170.

5. ASTM C1202. Standard test method for electrical indication of concrete's ability to resist chloride ion penetration. West Conshohocken, Pennsylvania: ASTM International; 2005.

6. Claisse PA, Elsayad $\mathrm{H}$ and Ganjian E. Modelling the rapid chloride permeability test. Cement and Concrete Research. 2009; 40(30):405-409.

7. Lizarazo-Marriaga $\mathbf{J}$ and Claisse P. Effect of the non-linear membrane potential on the migration of ionic species in concrete. Electrochimica Acta. 2009; 54(10):2761-2769.

8. Bockris J and Reddy A. Modern electrochemistry. 1 Ionics. New York: Plenum Press; 1998.

9. Lizarazo-Marriaga J and Claisse P. Determination of the chloride transport properties of blended from a new electric migration test. Concrete in aggressive aqueous environments - Performance, testing, and modelling. RILEM Publications; 2009.

10. Lizarazo-Marriaga J and Claisse P. Determination of the concrete chloride diffusion coefficient based on an electrochemical test and an optimization model. Materials Chemistry and Physics. 2009; 117:536-543.

11. Lizarazo-Marriaga $J$ and Claisse P. Determination of the transport properties of a blended concrete from its electrical properties measured during a migration test. Magazine of Concrete Research. 2010; 60(3):163-175.

12. Prince W, Perami R and Espagne M. Mechanisms involved in the accelerated test of chloride permeability. Cement and Concrete Research. 1999; 29(5):687-694. 